\title{
Observation of Both Second-Harmonic and Multiphoton-Absorption-Induced Luminescence in $\mathrm{ZnO}$
}

\author{
D. C. Dai, S. J. Xu, S. L. Shi, M. H. Xie, and C. M. Che
}

\begin{abstract}
The confusion over second-harmonic generation (SHG) and multiphoton-absorption (MPA)-induced luminescence in $\mathrm{ZnO}$ is observed under excitation with femtosecond laser between 800 and $1000 \mathrm{~nm}$ at room temperature. Excitation power dependence measurements show SHG is a two-photon process, and MPA is a three- or four-photon process. These are confirmed with interferometric pulse autocorrelation experiments. Excitation power density is a main regulatory factor for this confusion.
\end{abstract}

Index Terms-Interferometric autocorrelation, luminescence, multiphoton absorption (MPA), second-harmonic generation (SHG), zinc oxide ( $\mathrm{ZnO})$.

$\mathbf{R}$ ECENTLY, $\mathrm{ZnO}$ has attracted increasing interest as a promising wide bandgap optoelectronic material in the ultraviolet (UV) band for applications as laser diode and light-emitting diode operating at room temperature. Beyond the optical properties induced by UV laser [1], its nonlinear optical properties induced by visible and near-infrared lasers have drawn much attention in past years. For example, second(SHG) or third-harmonic generation [2]-[9], multiphoton absorption (MPA) [10]-[12], and MPA-induced luminescence [13]-[15]. Of particular interest are the nonlinear properties at the half bandgap spectral range [3], [6], [8], [9], [12], [14], [15]. These are all significant not only for understanding the basic physical properties but also for the development of applications in nonlinear optoelectronics.

Under excitation with femtosecond lasers at $\sim 800 \mathrm{~nm}$ $(1.55 \mathrm{eV})$, slightly below the half bandgap of $\mathrm{ZnO}(\mathrm{Eg} / 2=$ $1.70 \mathrm{eV}$ ), the papers [3], [6], [8], and [9] reported only the SHG process in different $\mathrm{ZnO}$ materials; however, other work ([12] and [14]) discussed only the two-photon absorption (2PA) process in $\mathrm{ZnO}$ films. In other words, by applying similar excitation lasers $\left(\lambda_{E}\right)$ to similar materials, completely different results are observed. Clearly it seems that the two bodies of work are contradictory.

Manuscript received January 3, 2006; revised April 9, 2006. This work was supported by Hong Kong RGC CERG under Grant HKU-7036/03P, and by the University of Hong Kong research Grant 10205780.

D. C. Dai was with the University of Hong Kong, Hong Kong. He is now with the Department of Physics, Durham University, Durham DH1 3LE, U.K. (e-mail: dechang.dai@durham.ac.uk).

S. J. Xu, S. L. Shi, and M. H. Xie are with the Department of Physics and HKU-CAS Joint Laboratory on New Materials, the University of Hong Kong, Hong Kong (e-mail: sjxu@hkucc.hku.uk).

C. M. Che is with the Department of Chemistry and HKU-CAS Joint Laboratory on New Materials, the University of Hong Kong, Hong Kong.

Digital Object Identifier 10.1109/LPT.2006.877620

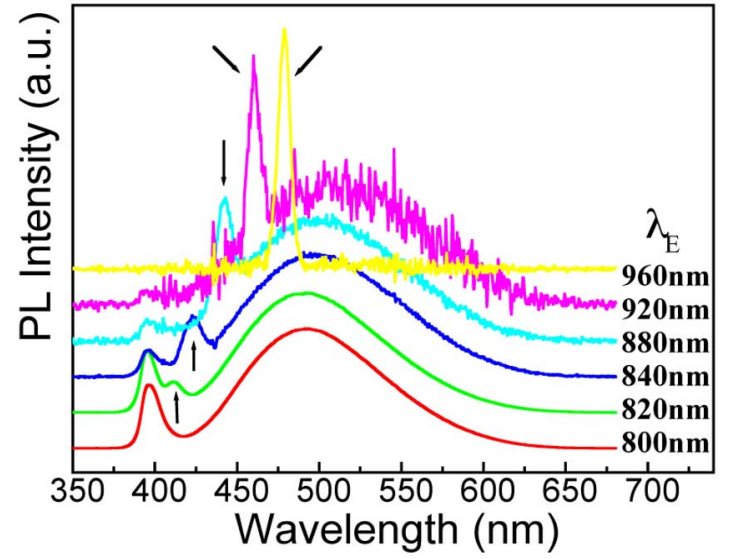

Fig. 1. MPA-induced luminescence spectra of $\mathrm{ZnO}$. (Color version available online at http://ieeexplore.ieee.org.)

In this letter, we discuss our observations on the confusion over SHG and MPA processes at the three-photon absorption band of $\mathrm{ZnO}\left(1000 \mathrm{~nm}>\lambda_{E} \geq 800 \mathrm{~nm}\right)$. Two distinct processes are distinguishable using excitation power dependence measurements, and with femtosecond interferometric pulse autocorrelation experiments. The SHG is a two-photon process: it always has a quadratic relationship with excitation power; yet the MPA is a three- or four-photon process: it follows the cubic or higher order power law. The excitation power density is a main regulatory factor for this confusion. And, the interferometric pulse autocorrelation of four-photon absorption is demonstrated for the first time.

The sample used in our experiments is a wurtzite structure $\mathrm{ZnO}$ single crystal with a thickness of $1 \mathrm{~mm}$. The femtosecond laser source is a self-mode-locked Ti:sapphire oscillator (Tsunami), delivering $\sim 80-\mathrm{fs}$ pulses at $82 \mathrm{MHz}$ and is tunable between 700 and $1000 \mathrm{~nm}$; the pulse spectrum is near Gaussian shape, and its bandwidth is $\sim 20 \mathrm{~nm}$. The excitation laser is focused onto the sample at near normal incidence with a $20-\mathrm{cm}$ focal mirror. Backward luminescence from the sample is recoded with a spectrometer and a photomultiplier tube. The power of excitation laser is controlled with a neutral density filter. The interferometric pulse autocorrelation experiments are carried out with a Michaelson-type interferometer [16]. Other details of the experiments are presented in [15]. All of experiments are performed at room temperature.

Fig. 1 shows the representative femtosecond laser MPA-induced luminescence spectra of $\mathrm{ZnO}$ excited at different wavelengths. The narrow UV band at $395 \mathrm{~nm}(3.14 \mathrm{eV})$ and broad vis- 

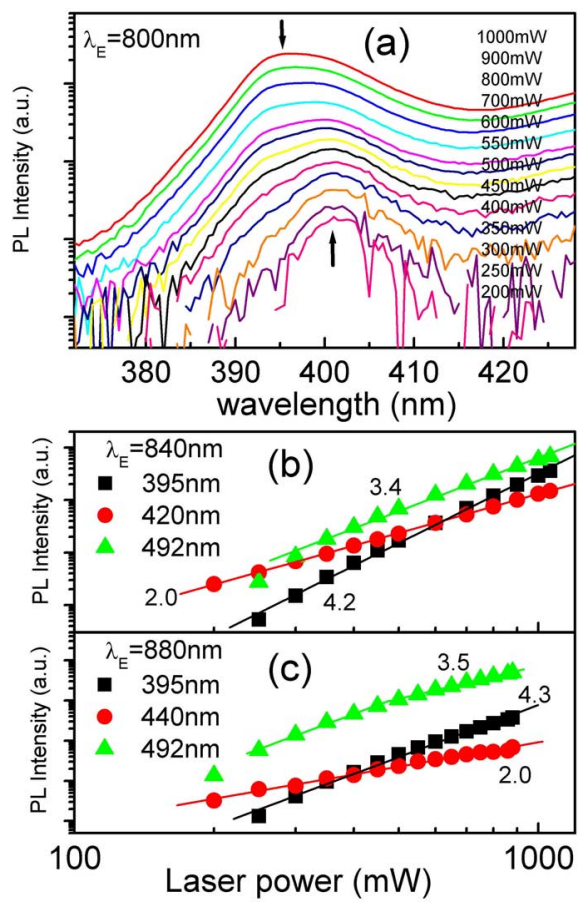

Fig. 2. (a) Excitation power-dependent luminescence spectra of $\mathrm{ZnO}$ for $\lambda_{E}=$ $800 \mathrm{~nm}$; (b) and (c) Excitation power dependence of SHG and luminescence of $\mathrm{ZnO}$ for $\lambda_{E}=840 \mathrm{~nm}$ and $\lambda_{E}=880 \mathrm{~nm}$, respectively. The data for $395 \mathrm{~nm}$ are reproduced from [15], the solid lines are fitting results. (Color version available online at http://ieeexplore.ieee.org.)

ible band centered at $\sim 492 \mathrm{~nm}(2.52 \mathrm{eV})$ are luminescent emissions of $\mathrm{ZnO}$; their relative intensities and the related photophysical processes under such multiphoton excitation have been discussed in [15]. The luminescence of $\mathrm{ZnO}$ becomes weak as $\lambda_{E}>900 \mathrm{~nm}$, and can hardly be detected by our system as $\lambda_{E}>960 \mathrm{~nm}$ because the threshold for multiphoton excitation is close to the maximum output power of our laser.

The SHG emission peaks of the excitation laser are marked by arrows in Fig. 1. They always overlap with certain spectral components of luminescent spectrum of $\mathrm{ZnO}$, and are often identified as shoulders as $880 \mathrm{~nm}>\lambda_{E}>800 \mathrm{~nm}$ because the bandwidth of visible luminescence $(\sim 100 \mathrm{~nm})$ is much broader than that of the SHG emission $(\sim 15 \mathrm{~nm})$. The SHG emission peaks are easy to resolve as $\lambda_{E}>900 \mathrm{~nm}$ as the MPA-induced luminescence in $\mathrm{ZnO}$ becomes weak. The SHG emission is difficult to observe as $\lambda_{E}<800 \mathrm{~nm}$ because it falls into the strong linear absorption band of cross bandgap transition of $\mathrm{ZnO}$. The mechanism of the SHG process in different $\mathrm{ZnO}$ materials has been discussed extensively [2]-[9].

To further elucidate the apparent confusion between MPAinduced luminescence and SHG emission in $\mathrm{ZnO}$, excitation power dependence of three emission bands for each $\lambda_{E}$ has been measured, the representative results are depicted in Fig. 2 on logarithmic scale. Fig. 2(a) shows the excitation power-dependent luminescence spectra for $\lambda_{E}=800 \mathrm{~nm}$. The peak position of the UV band shifts gradually from $400 \mathrm{~nm}$ at lower excitation power to $395 \mathrm{~nm}$ at higher power. Actually, this UV band is a result of superposition of MPA-induced UV luminescence $(395 \mathrm{~nm})$ and SHG emission $(400 \mathrm{~nm})$; two peaks are hard to resolve by deconvolution in a single luminescent spectrum as both the wavelength and bandwidth of the peaks are so close. The SHG is a strict two-photon process and always has a quadratic relationship with excitation power, yet the MPA is a resonant three-photon process at $\lambda_{E}=800 \mathrm{~nm}$ and follows a cubic power law; hence, the intensity of the component at $395 \mathrm{~nm}$ grows much faster than that at $400 \mathrm{~nm}$ as the excitation power is increased. As a result, the UV component at $395 \mathrm{~nm}$ dominates the spectra at higher excitation power. When the excitation power is lower than the threshold for MPA, the MPA-induced luminescence is very weak, SHG emission dominates the UV band, and the peak position is $400 \mathrm{~nm}$.

Fig. 2(b) and (c) plot the intensities of the three deconvolved emission bands as a function of excitation laser power for $\lambda_{E}=$ $840 \mathrm{~nm}$ and $\lambda_{E}=880 \mathrm{~nm}$, respectively. The slopes obtained from fitting are 2.0, 4.2, and $~ 3.4$ for SHG emission, MPAinduced UV, and visible luminescence, respectively. The data from other $\lambda_{E}$ give similar results. These immediately prove that SHG is a two-photon process, and MPA is a three- or fourphoton process. The deviation of the slope for the three-photon process from 3.0 to 4.2 is attributed to the involvement of the deep centers as intermediate states in the multiphoton transition, which makes the MPA transition responsible for UV luminescence a four-photon process [15].

Generally, the power density threshold for a three-photon process is higher than that for a two-photon process by at least one order of magnitude for certain $\lambda_{E}$, hence, the SHG emission in $\mathrm{ZnO}$ can be generated efficiently at lower excitation power as $1000 \mathrm{~nm}>\lambda_{E}>800 \mathrm{~nm}$, MPA requires higher excitation power. These are obvious in Fig. 2. Under normal laboratory conditions with nanosecond lasers, the power density is only sufficient for studying the SHG process in $\mathrm{ZnO}$ [2], [4], [5]; however, the use of femtosecond lasers allows for higher excitation power density $\left(>1 \mathrm{GW} / \mathrm{cm}^{2}\right)$; for example in [12] and [14] 2PA in $\mathrm{ZnO}$ is clearly detected through the use of tightly focused femtosecond laser even with a microscope objective. When excitation power density is properly controlled, femtosecond lasers can also be used to generate SHG emission in $\mathrm{ZnO}$ [3], [6], [8], [9], yet care must be taken to avoid the confusion from MPA-induced luminescence.

The interferometric pulse autocorrelation technique is frequently used for quantitative characterization of the multiphoton process and ultrashort laser pulse [16]. The signal background ratio of the autocorrelation trace is an indicator of the order of certain MPA processes: the ratios for two-, three- and four-photon processes are $8: 1,32: 1$, and $128: 1$, respectively [15]-[17]. To further investigate the confusion between SHG- and MPA-induced luminescence in $\mathrm{ZnO}$, the interferometric autocorrelation experiments are carried out for each $\lambda_{E}$ while the SHG emission, UV, and visible luminescence are being monitored. The representative results are plotted in Fig. 3. The signal background ratios obtained from UV traces range from $45: 1\left(\lambda_{E}=810 \mathrm{~nm}\right)$ to $128: 1\left(\lambda_{E}>900 \mathrm{~nm}\right)$, those from visible traces are from $25: 1\left(\lambda_{E}=800 \mathrm{~nm}\right)$ to $45: 1$ $\left(\lambda_{E}=960 \mathrm{~nm}\right)$. These ratios indicate the MPA responsible for UV luminescence is mainly a four-photon process, and the MPA responsible for visible luminescence is a three-photon process. The ratios from the SHG emission are always close to 

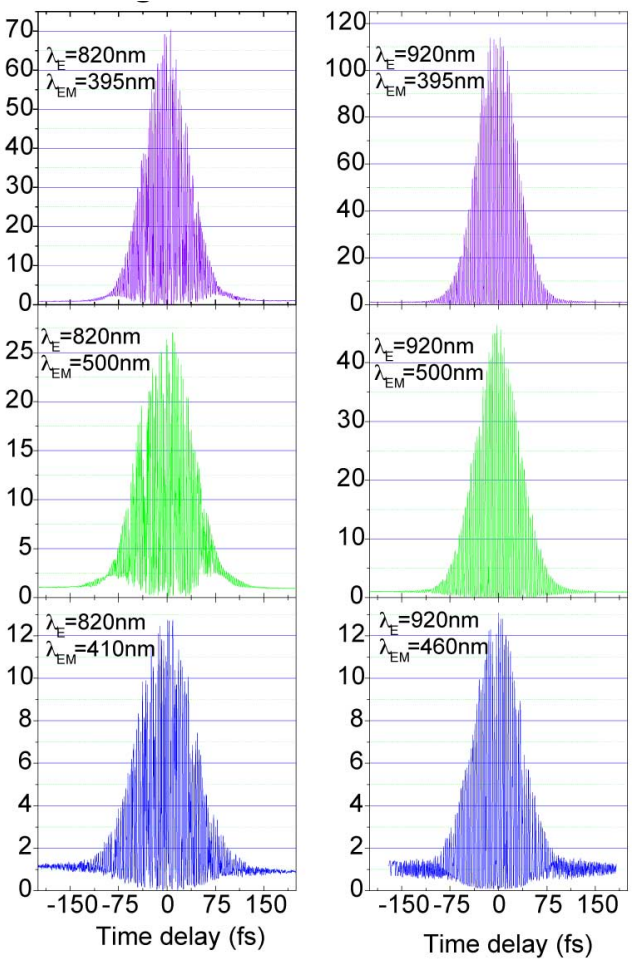

Fig. 3. Interferometric pulse autocorrelation traces for $\lambda_{E}=820 \mathrm{~nm}$ and $\lambda_{E}=920 \mathrm{~nm}$. The backgrounds are normalized to 1 for all traces. (Color version available online at http://ieeexplore.ieee.org.)

$8: 1$, but slightly larger ( $\sim 13: 1$, see Fig. 3$)$; this is because the SHG signal recorded in detection contains a small contribution from the MPA-induced luminescence. The results of these experiments are consistent with the excitation power dependences in Fig. 2. To our knowledge, this is the first report of an interferometric autocorrelation trace with a ratio of $128: 1$.

By the fundamentals of nonlinear optics, the SHG efficiency is determined by microscopic symmetry of material structure and the incident angle of laser beam [3]-[5]; additionally, the visible luminescent band of $\mathrm{ZnO}$ is tightly related to the level of defect states [15]. To achieve full understanding of the confusion, further experiments on $\mathrm{ZnO}$ crystals and films with different defects level are necessary. The perfect $\mathrm{ZnO}$ crystal does not contain defect states, hence, it has no visible luminescence, so the confusion does not exist as $\lambda_{E}>820 \mathrm{~nm}$. However, it is still a challenge at present to make such a high-quality $\mathrm{ZnO}$ material because the bandgap is too broad to avoid the inclusion of any defects.

In summary, we have studied the relationship between SHGand MPA-induced luminescence in $\mathrm{ZnO}$ with a femtosecond laser between 800 and $1000 \mathrm{~nm}$. The excitation power density is a main regulatory factor for this confusion; it can be used to selectively control SHG or MPA process in ZnO. The interferometric autocorrelation for four-photon absorption is demonstrated for the first time. This letter is instructive in the development of nonlinear optical applications of $\mathrm{ZnO}$.

\section{REFERENCES}

[1] B. Guo, Z. R. Qiu, and K. S. Wong, "Intensity dependence and transient dynamics of donor-acceptor pair recombination in $\mathrm{ZnO}$ thin films grown on (001) silicon," Appl. Phys. Lett., vol. 82, pp. 2290-2292, 2003.

[2] H. Cao, J. Y. Wu, H. C. Ong, J. Y. Dai, and R. P. H. Chang, "Second harmonic generaton in laser ablated zinc oxide thin films," Appl. Phys. Lett., vol. 73, pp. 572-574, 1998.

[3] U. Griebner, R. A. Kaindl, T. Elsaesser, and W. Seeber, "Frequency doubling and autocorrelation studies of 20-fs pulses using polycrystalline zinc oxide thin films," Appl. Phys. B, vol. 67, pp. 757-760, 1998.

[4] G. Wang, G. K. L. Wong, and J. B. Ketterson, "Redetermination of second-order susceptibility of zinc oxide single crystals," Appl. Opt., vol. 40, pp. 5436-5438, 2001.

[5] G. Wang, G. T. Kiehnie, G. K. L. Wong, J. B. Ketterson, X. Liu, and R. P. H. Chang, "Large second hamonic response in $\mathrm{ZnO}$ thin films," Appl. Phys. Lett., vol. 80, pp. 401-403, 2002.

[6] J. C. Johnson, H. Q. Yan, R. D. Schaller, P. B. Peterson, P. D. Yang, and R. J. Saykally, "Near-field imaging of nonlinear optical mixing in single zinc oxide nanowires," Nano. Lett., vol. 2, pp. 279-283, 2002.

[7] G. I. Petrov, V. Shcheslavskiy, V. V. Yakovlev, I. Ozerov, E. Chelnokov, and W. Marine, "Efficient third-harmonic generation in a thin nanocrystal-line film of ZnO," Appl. Phys. Lett., vol. 83, pp. 3993-3395, 2003.

[8] U. Neumann, R. Grunwald, U. Griebner, G. Stainmeyer, and W. Seeber, "Second-harmonic efficiency of $\mathrm{ZnO}$ nanolayers," Appl. Phys. Lett., vol. 84, pp. 170-173, 2004.

[9] T. Scheidt, E. G. Rohwer, H. M. von Bergmann, and H. Stafast, "Optical second harmonic imaging: A versatile tool to investigate semiconductor surfaces and interfaces," Eur. Phy. J., Appl. Phys., vol. 27, pp. 393-397, 2004

[10] I. M. Catalano, A. Cingolani, and M. Lepore, "Two-photon absorption spectra of direct and indirect materials: $\mathrm{ZnO}$ and AgCl," Phys. Rev. B, vol. 33, pp. 7270-7273, 1986.

[11] J. Wrzesinski and D. Frohlich, "Two-photon and three-photon spectroscopy of $\mathrm{ZnO}$ under uniaxial stress," Phys. Rev. B, vol. 56, pp. 13087-13093, 1997.

[12] J. H. Lin, Y. J. Chen, H. Y. Lin, and W. F. Hsieh, "Two-photon resonance assisted huge nonlinear refraction and absorption in $\mathrm{ZnO}$ thin films," Appl. Phys., vol. 97, p. 33526, 2005.

[13] J. Collet and T. Amand, "Picosecond spectroscopy of excitons and biexcitons in $\mathrm{ZnO}$ at high density," Phys. Rev. B, vol. 33, pp. 4129-4135, 1986.

[14] X. M. Wen, P. Xu, and P. B. Lukins, "Characterization of enhanced emission from excimer laser treated $\mathrm{ZnO}$ ceramics using one- and two-photon luminescence spectroscopy and microcopy," J. Lumin., vol. 106, pp. 1-7, 2004.

[15] D. C. Dai, S. J. Xu, S. L. Shi, M. H. Xie, and C. M. Che, "Efficient multiphoton-absorption-induced luminescence in single-crystalline $\mathrm{ZnO}$ at room temperature," Opt. Lett., vol. 30, pp. 3377-3379, 2005.

[16] J.-C. Diels and W. Rudolph, Ultrashort Laser Pulse Phenomena: Fundamentals, Techniques, and Applications on a Femtosecond Time Scale.. New York: Academic, 1996, ch. 8.

[17] P. Langlois and E. P. Ippen, "Measurement of pulse asymmetry by three-photon-absorption autocorrelation in a GaAsP photodiode," Opt. Lett, vol. 24, pp. 1868-1870, 1999. 